\title{
Study of QPSK Modulator and Demodulator in Wireless Communication System Using MATLAB
}

\author{
http://dx.doi.org/10.3991/ijim.v7i2.2239 \\ Sharief Nasr Abdel-Razeq, Areej Salah Al-Azzeh, Rawan Yousef Ayyoub \\ Al-Balqa' Applied University, Irbid, Jordan
}

\begin{abstract}
Quadrature Phase-Shift Keying (QPSK) modulation technique is one of the most widely used modulation scheme in modern digital communication system as it provides high bandwidth efficiency. In this paper, the model of QPSK modulator and demodulator has been simulated using MATLAB Simulink.
\end{abstract}

Index Terms-BER, Demodulator, Eye pattern, MATLAB, Modulator, QPSK.

\section{INTRODUCTION}

Communications systems can be defined as transferring data from one place to another; it is done in wired or wireless manners. In either ways; we have to modulate the baseband signal to make it suitable for transmission over the medium. This process called Modulation which could be Analog or Digital Modulation.

In the last years, a major transition from analog to digital modulation techniques has occurred and it can be seen in all areas of satellite communications systems, cellular and wireless [1].

Digital modulation is a process that impresses a digital symbol onto a signal suitable for transmission over a wired or wireless medium in order to receive that signal at receiving end correctly without any loss of information [2]. QPSK is one of the most popular digital modulation techniques used for satellite communications and sending data over cable networks, videoconferencing, cellular phone systems, and other forms of digital communication over Radio Frequency (RF) carrier [3].

The Quadrature Phase Shift Keying (QPSK) is one of the most used Phase Shift Keying digital modulations, due to its simplicity, excellent power and bandwidth efficiency [4].QPSK allows the signal to carry twice as much information as ordinary PSK 'or BPSK' using the same bandwidth.

In practice, engineering problems are difficult to solve. Most often, numerical methods are used as analytical solutions to such problems. However, numerical methods themselves are usually iterative in nature requiring several intermediate steps in order to arrive at a solution. This means much time may be months or years, will be needed and more energy expended in an effort to solve a particular problem. In order to lessen these difficulties, there is a need to develop computer algorithms that can solve the problem at hand [5].

Such problems can now readily be solved in a few hours by the use of commercial engineering software packages, such as MATLAB/SIMULINK $®$, ANSOFT ${ }^{\circledR}$, SIMPLORER $\AA$, WORKBENCH ${ }^{\circledR}$, etc. [5].

MATLAB is considered to be a good choice to perform our study because of its programming capability which allows user-developed functions. Moreover, there are several optional toolboxes written for special applications such as signal processing, control systems design, system identification, statistics, neural networks, fuzzy logic, symbolic computations, and others. MATLAB has been enhanced by the very powerful SIMULINK program [6].

\section{MATLAB SIMULINK MODELS AND SIMULATION RESULTS}

QPSK can be generated from two orthogonal BPSK modulated signals (In-phase component and Quadraturephase component). So, the model of QPSK modulator and demodulator has two branches, the upper branch presents the In-phase component and the lower branch presents Quadrature-phase component.

\section{A. The QPSK Modulator Model and its Result}

The QPSK Modulator was simulated in Matlab using Simulink tool boxes. The simulation model is given by Figure 1.

The simulation results appeared as shown in the Figure 2 below. It's worth mentioning that these results were taken at the output of "Outputs QPSK Transmitter" block.

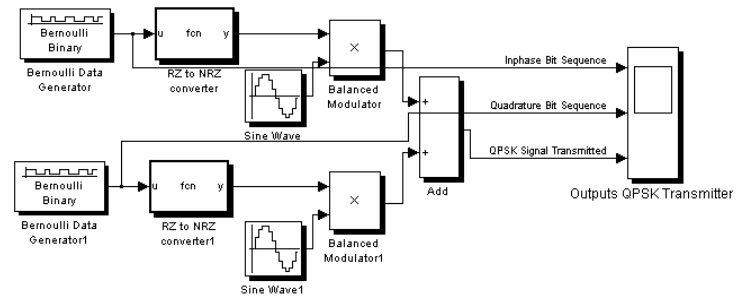

Figure 1. QPSK Modulator Simulink model

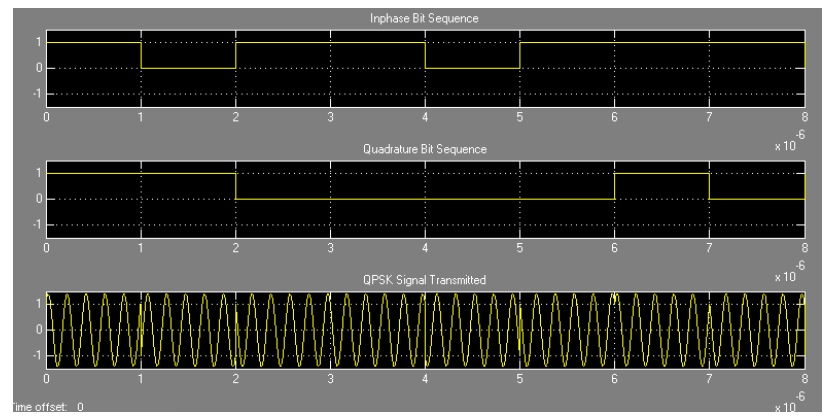

Figure 2. The result of QPSK Modulator 
B. The QPSK Demodulator Model and its Result.

The QPSK Demodulator was simulated in Matlab using Simulink tool boxes. The simulation model is given by the Figure below:

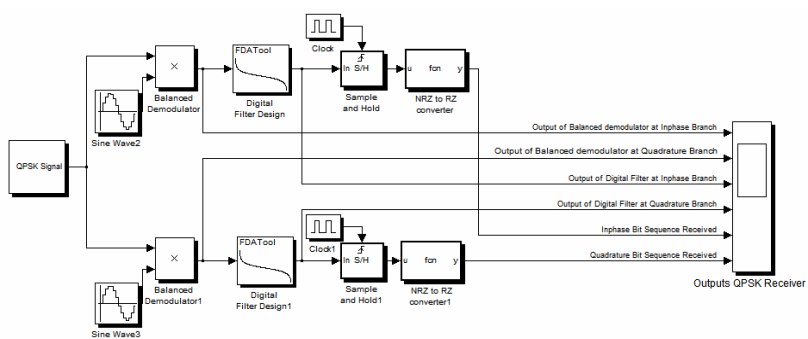

Figure 3. QPSK Demodulator

The simulation results appeared as shown in the Figure 4 below. It's worth mentioning that these results were taken at the output of "Outputs QPSK Receiver" block.

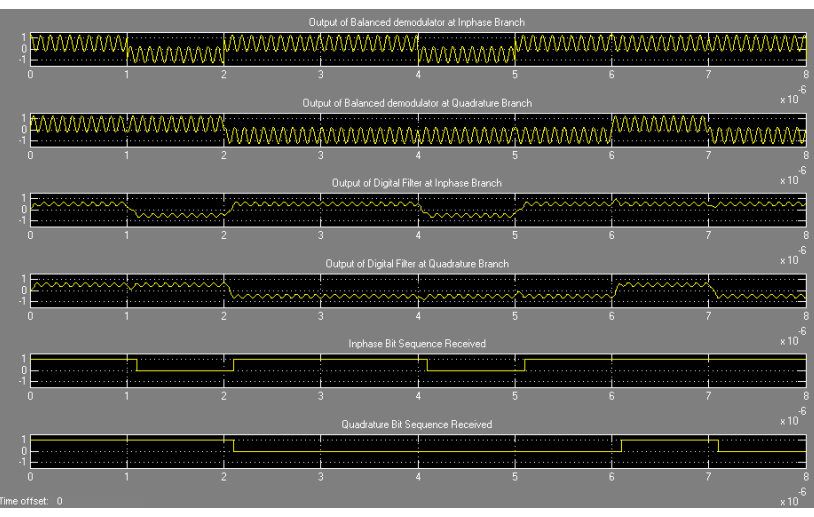

Figure 4. The result of QPSK Demodulator

\section{The QPSK Modulator and Demodulator Model and its Result.}

The third stage of our work was to connect QPSK Modulator with QPSK Demodulator directly without any external noise; and the overall system model is given by the Figure below:

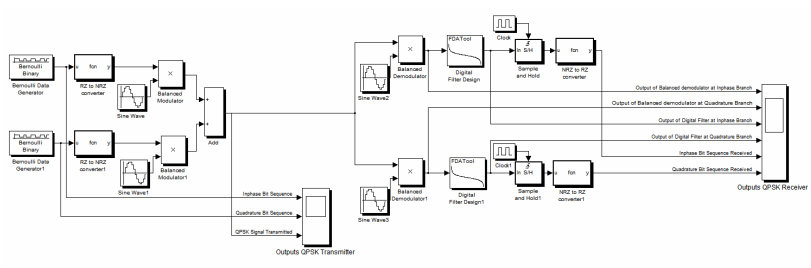

Figure 5. Fi QPSK Modulator and Demodulator

The results of the QPSK Modulator at "Outputs QPSK Transmitter" block are shown below:

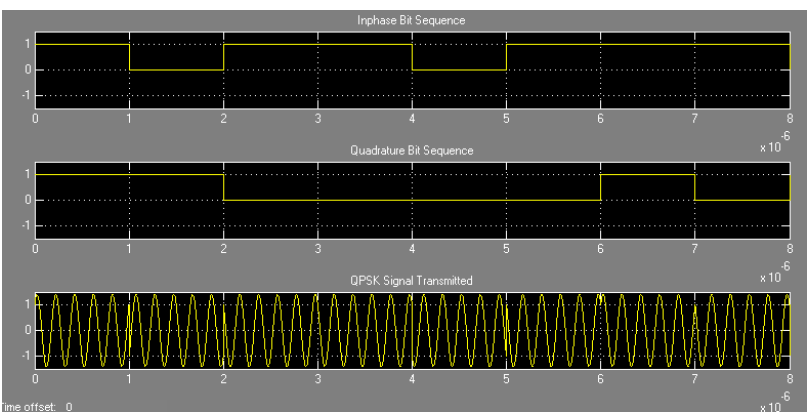

Figure 6. .The result of QPSK Modulator
The results of the QPSK Demodulator at "Outputs QPSK Receiver" block are shown below:

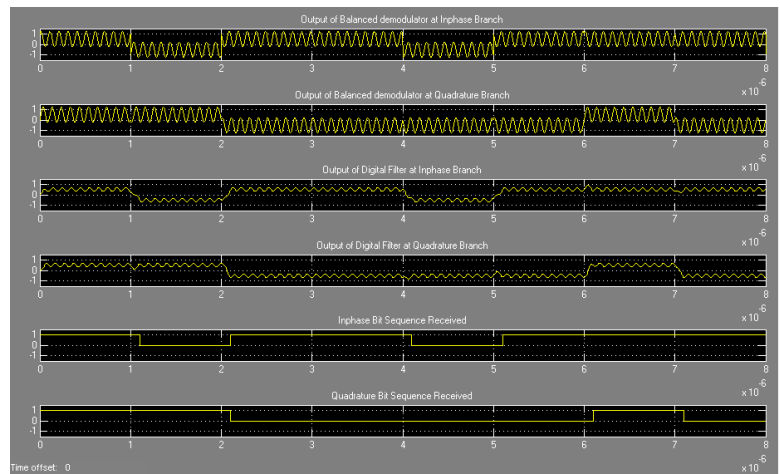

Figure 7. The result of QPSK Demodulator

\section{The QPSK Modulator and Demodulator under} AWGN Channel and its Result.

After we have seen the connection of overall QPSK system and its results under noise-free channel, its worth to see the results under Additive White Gaussian Noise (AWGN) channel, the Simulink model became as shown below:

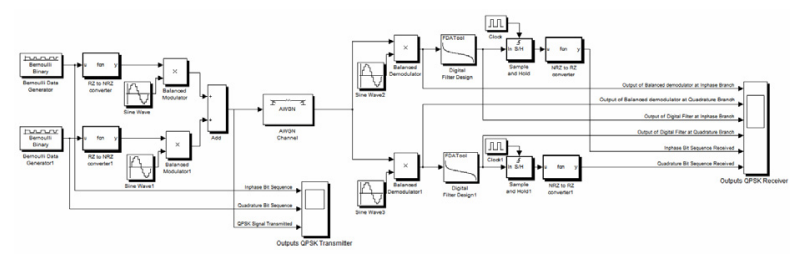

Figure 8. QPSK Modulator and Demodulator under AWGN Channel

Under AWGN channel, $\frac{\mathbf{E}_{\mathbf{b}}}{\mathbf{N}_{0}}$ is the "controlling parameter" which decides the amount of noise added by that ter" $\mathbf{E}_{\mathbf{b}}$ channel. Hence, we have taken different values of $\overline{\mathbf{N}_{0}}$ and then we compared the results. The different values are shown below:

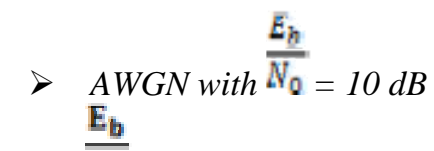

For $\overline{\mathrm{N}_{0}}$ of $10 \mathrm{~dB}$; the results appeared as shown Figure 9below:

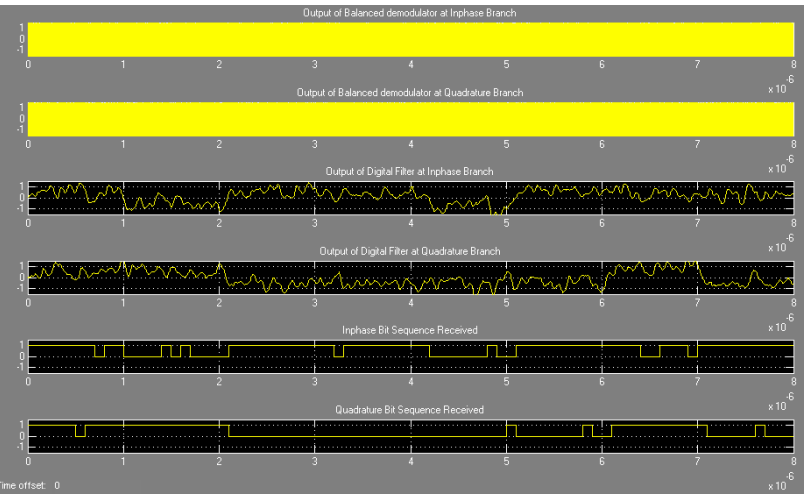

Figure 9. .The result of QPSK Demodulator under AWGN Channel with $10 \mathrm{~dB} \frac{\underline{E_{t}}}{N_{a}}$ 
$>A W G N$ with $\frac{E_{b}}{N_{0}}=16 d B$

The second value was $\frac{\mathbf{E}_{\mathbf{b}}}{\mathbf{N}_{0}}=16 \mathrm{~dB}$. The results appeared as shown in Figure 10 below:

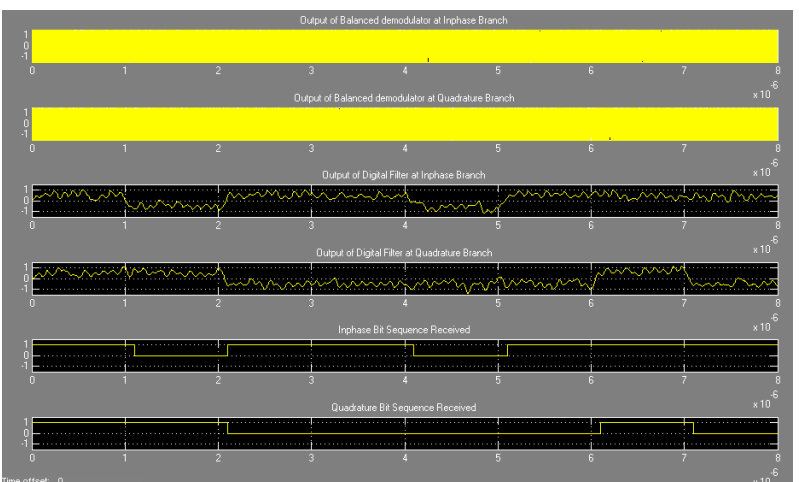

Figure 10. The result of QPSK Demodulator under AWGN Channel

$$
\text { with } 16 \mathrm{~dB} \underline{\underline{E_{z}}}
$$

It can be noticed that the performance of QPSK system is affected significantly under AWGN channel compared to noise-free channel. Moreover, the output waveform at the QPSK Demodulator affected severely when the value of $\frac{\mathbf{E}_{\mathbf{b}}}{\mathbf{N}_{0}}$ is decreased.

\section{E. The Constellation Diagram of QPSK Model and its Result.}

A constellation diagram shows the symbol locations in complex signal space. The horizontal axis is the real or Inphase component, which is also the amplitude of the cosine portion of the Quadrature carrier. The vertical axis is the imaginary or Quadrature component, which is also the amplitude of the sine portion of the Quadrature carrier. The amplitude of a symbol is its distance from the origin. The phase angle of a symbol is its angular displacement from the positive horizontal axis [7].

The model of QPSK Modulator with "Discrete - time scatter plot scope" as shown below in Figure 11:

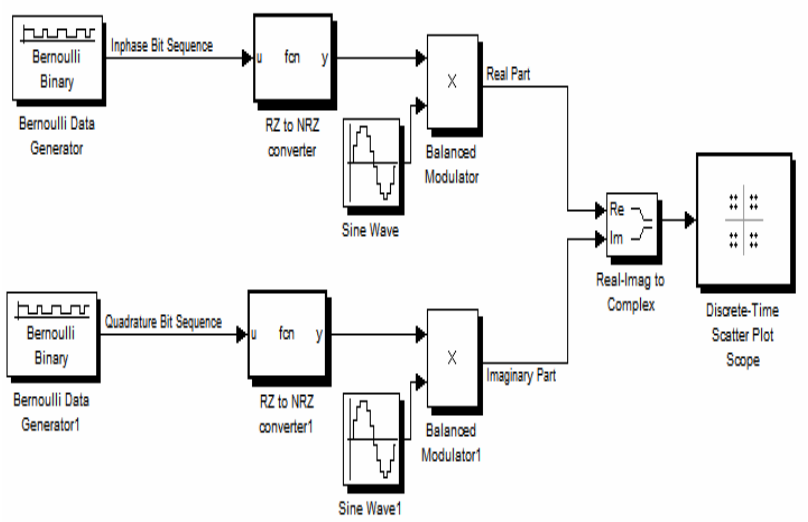

Figure 11. . The model of QPSK Modulator with Discrete - time Scatter plot scope

The result of "Discrete - time scatter plot scope" block appeared as shown below:

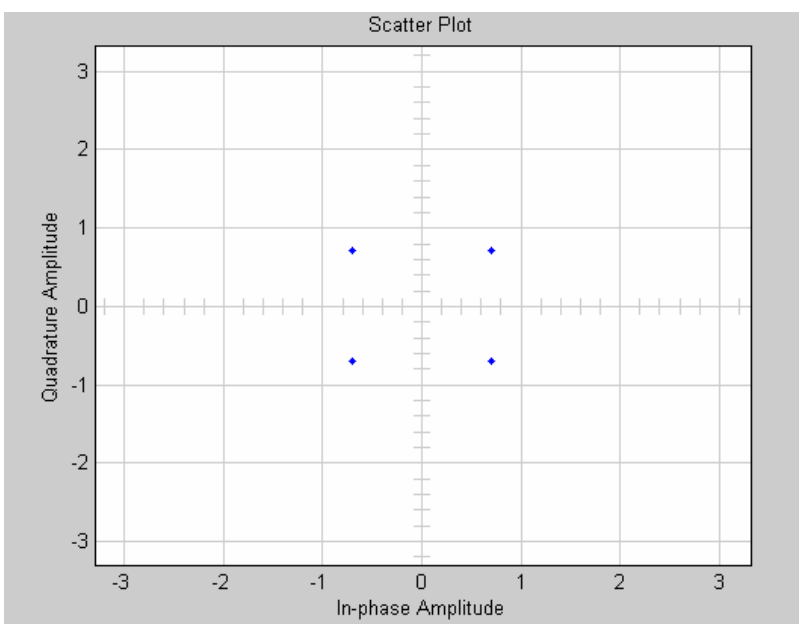

Figure 12. The result of Discrete - time Scatter plot scope at QPSK Transmitter

\section{F. An Eyepattern of QPSK Model and its Result.}

Eye pattern, also known as an eye diagram is a tool for studying the effects of Intersymbol Interference (ISI) and other channel impairments in digital transmission. The procedure to construct an Eyepattern is to plot the received signal against time on a fixed-interval axis [8].

The "Discrete-time eye diagram scope" was connected to the model as shown in Figure 13 below:

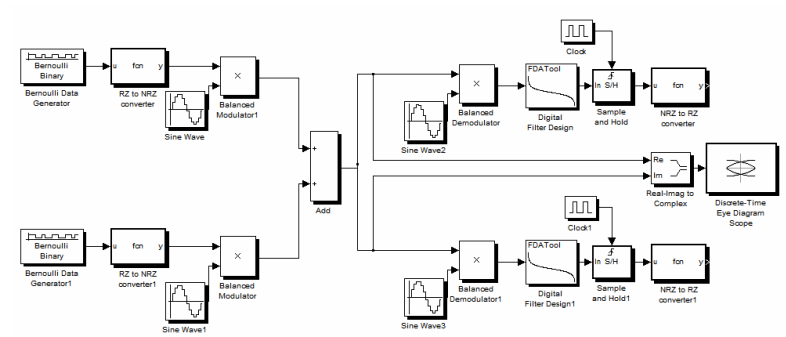

Figure 13. . The model of QPSK system with Discrete - time Eye diagram scope

The result of "Discrete - time eye diagram scope" appeared as shown below:

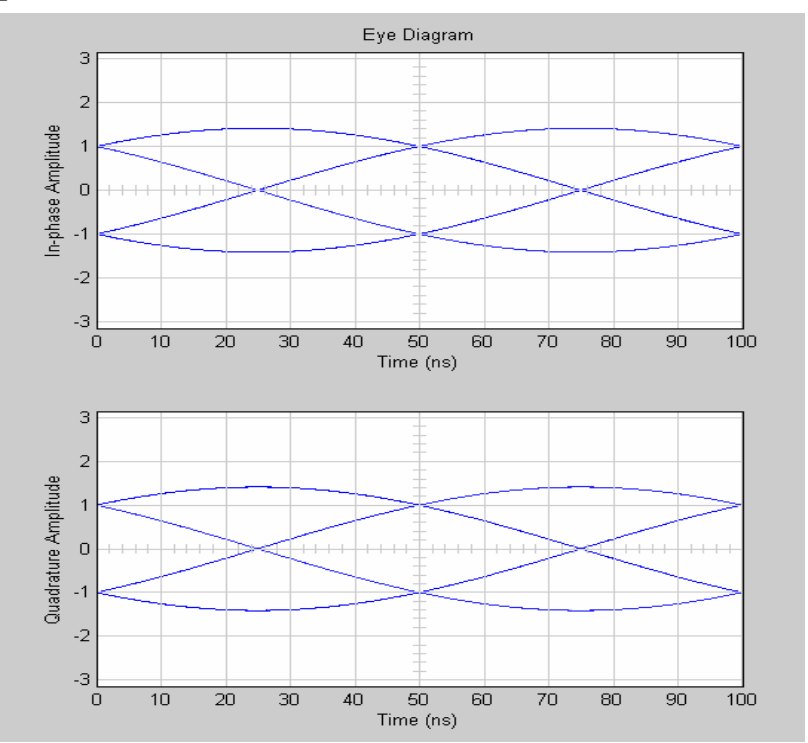

Figure 14. The result of Discrete - time Scatter plot scope at QPSK system 


\section{G. The Bit Error Rate of QPSK Model}

Bit Error Rate (BER) of a communication system is defined as the ratio of number of error bits and total number of bits transmitted during a specific period. It is the likelihood that a single error bit will occur within received bits, independent of rate of transmission [9].

The computer simulation is carried out by Monte Carlo method with the aid of MATLAB. The system in the simulation is subjected to AWGN channel [10].

The Bit Error Rate of QPSK Simulink Model as shown in Figure 15:

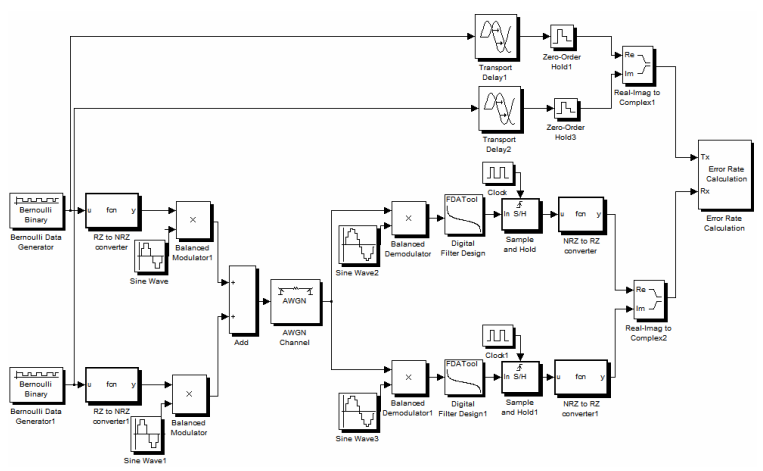

Figure 15. The model of QPSK system with Error Rate Calculation

The result of "Error - rate calculation" block appeared as shown below:

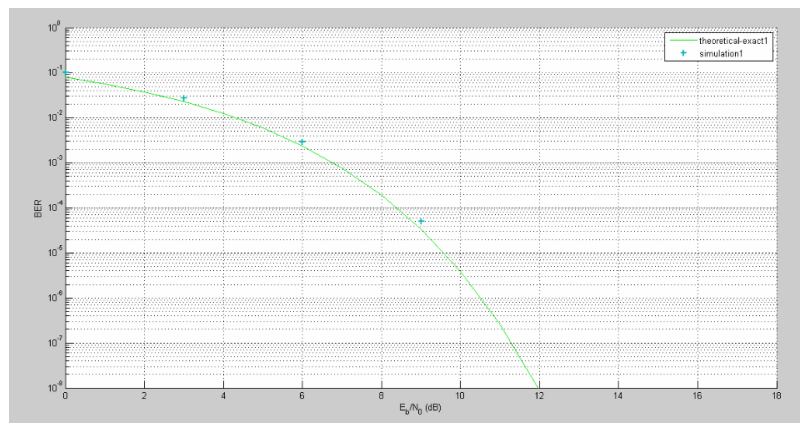

Figure 16. . The result of BERTool

\section{SIMULATION RESULTS DISCUSSION}

QPSK modulator and demodulator model was simulated using Matlab Simulink tool box as we mentioned earlier. We devoted this section to discuss the simulation results.

Firstly, QPSK modulator in Figure 1 has generated waveform signals at "Outputs QPSK Transmitter" scope; In-phase and Quadrature bit sequences which is the data. QPSK signal according to I and Q bit status was shown in Figure 2. It could be noted that the phases generated when $11,01,10$, and 00 were sent are $\pi / 4,3 \pi / 4,7 \pi / 4$, and $5 \pi / 4$, respectively. These results match the theoretical studies of QPSK.

In the other side, QPSK demodulator in Figure 3 received the QPSK signal and processed it to extract the Inphase and Quadrature data which were sent formerly. In Figure 4 the waveform signals which we get it on "Outputs QPSK Receiver" scope were showing the output of balanced demodulator, the output of digital filter, and the bit sequence received in each branch In-phase and Quadrature. We can note that the bit sequence in each branch is identical to the bit sequence we have sent.
After that, modulator and demodulator have been connected under noiseless channel as shown in Figure 5, the waveform signals resulted from modulator in Figure 6 are remained the same result in Figure 2, also the received bit sequences at In-phase and Quadrature branches in demodulator in Figure 7 matched the bit sequences at Inphase and Quadrature branches in modulator.

In Figure 8 the QPSK modulator and demodulator were connected under AWGN channel, The AWGN channel has influencing factor which is the $\frac{\underline{\mathbf{E}_{\mathrm{b}}}}{\mathrm{N}_{\mathrm{n}}}$, so we studied two cases, the first one is $\frac{\mathbf{E}_{\mathrm{b}}}{\mathbf{N}_{0}}$ equal to $10 \mathrm{~dB}$, with this value, the output generated from the demodulator has been affected by the noise added by AWGN channel as shown in Figure 9. It can be noticed that the effect appears at all waveform signals in demodulator in a form of mismatching between the bit sequences at In-phase and Quadrature branches between modulator and demodulator.

Moreover, when we increased $\frac{\mathbf{E}_{\mathbf{b}}}{\overline{\mathbf{N}_{0}}}$ to $16 \mathrm{~dB}$; which is the second case; the output of balanced demodulator and the output of digital filter in each branch were affected with noise. Yet, received bit sequences at In-phase and Quadrature branches in demodulator in Figure 10 matched the bit sequences at In-phase and Quadrature branches in modulator.

To get the constellation diagram of QPSK system, the "discrete-time scatter plot" scope was connected with QPSK modulator as shown in Figure 11 and the constellation diagram was as shown in Figure 12. We noticed that the constellation diagram of QPSK system contains four points forming a cycle, as well as these points satisfy the Gray code.

To check the effect of Intersymbol Interference for the system, we generated the eye pattern of our model using the "Discrete - time eye diagram" scope which was connected with QPSK demodulator as shown in Figure 13; we concluded from the eye pattern shown in Figure 14 that the eye in each branch In-phase and Quadrature branches has widely-opened eye.

The most important parameter in digital communication systems is BER, to generate the BER curve; we connected the circuit as shown in Figure 15. We noted from Figure 16 that the simulation points according to our system closed to the theoretical curve.

\section{CONCLUSIONS}

The main purpose of this paper was to study a complete model of one of the most commonly used modulation schemes in satellite communication systems, QPSK Modulator and Demodulator. It has been designed, simulated, and tested using MATLAB Simulink.

The key indicator of our model performance, such as Bit Error Rate (BER), constellation diagram, and eye Pattern, have been generated and analyzed. BER curve closed to the theoretical one, the constellation diagram had four points in one cycle which mean it has a constant envelope and this leads the system not to suffer from attenuation. Moreover, these points satisfy Gray code which provides minimum BER.

On the other hand, the model was not affected by Intersymbol Interference. This was observed by the opening eye in eye pattern. 


\section{REFERENCES}

[1] S. Popescu, A. Gontean, and D. Ianchis, "QPSK Modulator on FPGA," Applied Electronics Department, Faculty of Electronics and Telecommunications, Politehnica University of Timisoara, Romania, September, 2011

[2] S. Rakesh, P. Miguel, P. Valentín, C. Juan, and M. Javier, "Generation of Multi-Gigabit Per Second MQAM/MPSK-Modulated Millimeter Wave Carriers Employing Photonic Vector Modulator Techniques," Journal of light wave technology, vol. 25, November 2007.

[3] D. Michael, "QPSK Modulation and Error Correcting Codes," Agilent Technologies, Temple University, 2003.

[4] S. Mario and G. Dolecek, "Design and Simulation of QPSK Reconfigurable Digital Receiver," Department of Electronics, Institute INAOE, Puebla, México, 2010.

[5] O. Ogbonnaya, P. Govender, and E. Chikuni, "A new user-friendly Software for Teaching and Research in Engineering Education," International Conference and Exhibition on Power and Telecommunications-ICEPT, Nigeria, 2005.

[6] S. Hadi, "Introduction to MATLAB and Simulink," V3.0, Milwaukee, Wisconsin, 2000.
[7] G. James, "Digital Phase Modulation: A Review of Basic Concepts,” Chief Scientist Transcript International, Inc., 2003.

[8] M. Ian, "The role of Matlab and Simulink in teaching communications," Faculty of Electrical Engineering and Computers University of Porto, Portugal, 2009.

[9] V. Václav, "Error Performance of BPSK and QPSK in a Satellite Communications," Department of Electrical and Electronic Technology, FEEC, BUT, Czech Republic, 2005.

[10]Z. Sharef and A. Alaradi, "Performance Evaluation for WiMAX 802.16e OFDMA Physical Layer," Fourth International Conference on Computational Intelligence, Communication Systems and Networks, 2012. http://dx.doi.org/10.1109/CICSyN.2012.71

\section{AUTHORS}

Sharief Nasr Abdel-Razeq, Areej Salah Al-Azzeh, and Rawan Yousef Ayyoub are with Al-Huson University College, Al-Balqa' Applied University, Irbid, Jordan.

Received 12 August 2012. Published as resubmitted by the authors 20 March 2013. 